\title{
Modelling desiccation cracking in a homogenous soil clay layer: comparison between different hypotheses on constitutive behaviour
}

\author{
Cristina Jommi ${ }^{1, a}$, Niccolò Valimberti ${ }^{2}$, Roderick N.Tollenaar ${ }^{1}$, Gabriele Della Vecchia $^{2}$ and Leon A. van Paassen ${ }^{1}$ \\ ${ }^{1}$ Delft University of Technology, Department of Geoscience and Engineering, Stevinweg 1, 2628 CN Delft, The Netherlands \\ ${ }^{2}$ Politecnico di Milano, Department of Civil and Environmental Engineering, piazza Leonardo da Vinci 32, 20133 Milano, Italy
}

\begin{abstract}
Desiccation cracks are usually thought to start from the surface of an evaporating soil layer, and the available simplified models for crack initiation and propagation are based on this hypothesis. On the contrary, experimental results on a Dutch river clay showed that cracks in an evaporating soil layer may start and propagate below the surface, confirming earlier findings by other researchers. A simple one-dimensional model was set up to analyse the consequences of different hypotheses about the material behaviour on the crack onset in a homogenous soil layer undergoing surface drying. The results of the model show that dependence of the material behaviour on the rate of water content change is a necessary requirement for cracks to initiate below the surface. The conclusion suggests that, to properly understand cracking in an evaporating soil layer, an intrinsic time scale for the mechanical response must be accounted for, among all the other factors which were previously highlighted by other researchers. The key factor to predict crack onset below the surface is the dependence of the drying branch of the water retention curve of the compressible soil on the rate of drying, which would be justified by a rate dependent fabric evolution.
\end{abstract}

\section{Previous findings and motivation}

Cracks are a common occurrence in drying soil. They not only have effects on the integrity of geotechnical structures, but they also affect the hydraulic properties of the soil. The main driving mechanisms governing the occurrence of fractures in soil are the matric suction generated in the drying material and the internal mechanical constraints.

It is generally believed that fractures in soil start at the surface and then they propagate downwards. This is certainly the case in many situations. As described by Weinberger [1], under laboratory conditions the initiation of fractures commonly happens at the surface of the desiccating layer. However, it has been observed that it is not always the case. Evidence obtained from field observations [2] and experiments carried out on Dutch river clay in the laboratories of the Delft University of Technology and the Politecnico di Milano, indicate otherwise. During the tests, it was observed that the fractures can initiate and propagate under the surface.

Several simplified models have been created to simulate the generation of cracks in soil [3-6], but all of them are based on the hypothesis that the fracture initially starts at the top of the desiccating layer. The assumption is reasonable given that the dryer section in the soil profile is at the top, where the largest amount of suction is potentially developed. These simplified models tackle the drying process as a weakly coupled, nearly steadystate process. They use a unique relationship between the water content and the relevant variables playing a part in the mechanics of the system, and they cannot simulate cracks beginning below the surface.

It has been established experimentally that under the same environmental and initial conditions, thin clay layers dry faster than thick ones. They also exhibit less cracks, larger intact areas and wider fractures [7-12]. The phenomenon has been attributed to the difference between desiccation rates. This premise has been confirmed by studies performed by Tang et al [10-11] and Costa et al [12]. Corte and Higashi [7] also observed that the water contents at the onset of cracking decreased as the drying speed increased.

As experimental evidence shows, there is a clear rate dependency of the stresses involved during soil drying and cracking. Tamrakar et al [13] mentioned that there is also an increase in tensile strength with higher tensile pulling rates, although the trends and the magnitude of the change in the results do not seem to support such conclusion to a large extent. Actually, most of the results appear to indicate that there is no significant variation in the tensile strength with different pulling rates. This suggests that the key factor to predict crack onset below the surface must be looked for among the other hydro-mechanical properties governing the response of the compressible soil upon drying. This contribution presents an exercise aimed at analysing the consequences of different hypotheses about the material behaviour on the theoretical prediciton of crack initiation in a homogenous soil layer.

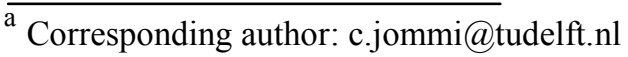




\section{Material and summary of the results}

An extensive investigation is being carried out at the Delft University of Technology to study the reuse of dredged clay sediments for civil applications. Part of this investigation focuses on the thorough understanding of how cracking occurs, since it is known to influence drainage, evaporation and ripening. The experimental study was performed on homogenous soil samples prepared in the laboratory, using a commercially available clay composed (by mass) of 50.2\% quartz, $21 \%$ vermiculite, $16.2 \%$ muscovite, $6.8 \%$ anorthite and $5.8 \%$ calcite approximately. The soil contains $3.1 \%$ sand, $54.9 \%$ silt and $42 \%$ clay. The liquid limit of the clay is $57 \%$, the plasticity index is $33 \%$, and the specific gravity is 2.74 . Reconstituted clay samples were prepared by mixing the commercial clay with water. The tested samples were prepared at different initial water contents.

At the Politecnico di Milano, thin vertical Perspex boards of different heights were filled with the clay in order to analyse the drying and crack formation along the vertical axis. Due to the stiffness contrast between the Perspex board and the clay, the first cracks always occurred at the side boundaries of the samples. After the first lateral cracks formed, the sample was almost free to shrink, with the base of the board offering little restraint to the shrinking process. Depending on the sample height and the evaporation rate, cracks were observed starting in the middle of the sample, even before any surface cracks appeared (Fig. 1).

At the Delft University of Technology, desiccation experiments were carried out by placing the clay at initial water contents between two and three times the liquid limit on wood, plastic and metal containers. The areas of the boxes ranged from 0.09 to $1 \mathrm{~m}^{2}$, and the initial soil thicknesses varied between 20 and $100 \mathrm{~mm}$. Detailed descriptions of the tests can be found in [14].

The clay behaviour was studied by a number of tests, which gave the following general conclusions:

(i) the tensile strength depends strongly on the water ratio, but it is almost insensitive to the rate of tensile strain;

(ii) the tensile stiffness decreases with increasing water ratio and tensile strain rate;

(iii) the air entry value upon drying depends on the initial water content, hence confirming the important role played by the soil fabric, but it is always in an order of magnitude between few to tens of $\mathrm{kPa}$.

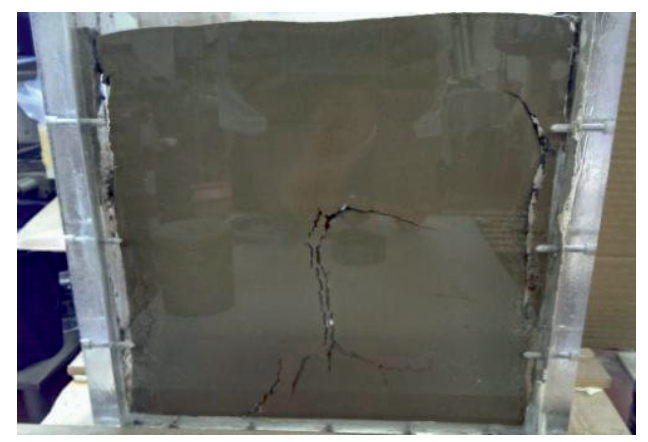

Figure 1. Evaporating clay slurry showing crack development below the surface in the clay body.

\section{A simple 1-D model of crack initiation}

In this section, reference is made to a homogenous isotropic deformable clay layer. Until the first crack appears, the layer can be described by a one-dimensional system with all relevant quantities varying only with depth, $z$. If the soil layer is initially saturated, previous research and results from the ongoing experimental investigation suggest that the first crack appears during the saturated state. Accepting this hypothesis, the water ratio $e_{w}=V_{w} / V_{s}$, where $V_{w}$ is the volume of water and $V_{s}$ is the reference volume of solids, is sufficient to describe the distribution of void ratio and water content throughout the soil profile before cracking occurs.

The water ratio profile changes in time and depth due to the evaporative flux at the top surface of the layer, and it was chosen as the driving variable of the hydromechanical model. The mechanical behaviour of the soil was described by the simplest allowable laws suggested by the experimental evidence. This was carried out to highlight the role played by different hypotheses in the response of the soil layer to the evaporation process. The hydraulic fluxes are not explicitly accounted for in the formulation, as the current water ratio distribution is sufficient for the suction profile to be calculated from the drying branch of the soil water retention curve (SWRC). In the formulation, compression stresses and strains are assumed positive. The change rate in the variables is designated by a superscript dot. The vertical and horizontal components of stress, $\sigma$, and strain, $\varepsilon$, are designated by subscripts $z$ and $h$, respectively.

\subsection{Relevant assumptions}

The soil is assumed to be homogenous, isotropic and saturated until the first crack occurs. If the variation of water content with depth and time is known, the current water ratio and water ratio rate can be calculated at any depth, serving as the input of the model.

A simple incremental hypo-elastic model is assumed to describe the material stiffness:

$$
\left[\begin{array}{c}
\dot{p}^{\prime} \\
\dot{q}
\end{array}\right]=\left[\begin{array}{cc}
K & 0 \\
0 & 3 G
\end{array}\right]\left[\begin{array}{c}
\dot{\varepsilon}_{v o l} \\
\dot{\varepsilon}_{d}
\end{array}\right]
$$

where $K$ is the (tangent) bulk modulus and $G$ is the (tangent) shear modulus. The stress and strain variables are defined as:

$$
\begin{aligned}
& p^{\prime}=\frac{1}{3}\left(\sigma_{z}^{\prime}+2 \sigma_{h}^{\prime}\right) \\
& q=\left(\sigma_{z}^{\prime}-\sigma_{h}^{\prime}\right) \\
& \varepsilon_{v o l}=\left(\varepsilon_{z}+2 \varepsilon_{h}\right) \\
& \varepsilon_{d}=\frac{2}{3}\left(\varepsilon_{z}-\varepsilon_{h}\right)
\end{aligned}
$$


The volumetric (shrinkage) strain rate is given by:

$$
\dot{\varepsilon}_{v o l}=-\frac{\dot{e}_{w}}{1+e_{w}}
$$

and it is assumed to be entirely due to a change in effective stress acting on the soil element:

$$
\dot{\varepsilon}_{v o l}=\frac{1}{K} \dot{p}^{\prime}==\frac{1}{K}\left(\dot{p}-\dot{u}_{w}\right)=\frac{1}{K}(\dot{p}+\dot{s})
$$

where $u_{w}$ is the pore water pressure and $s$ is the suction. Under saturated conditions, $s=-u_{w}$. It follows that the tangent bulk modulus can be obtained as the derivative of the SWRC, starting from the state at which positive suctions are measured:

$$
K=-\left(1+e_{w}\right) \frac{\partial s}{\partial e_{w}}
$$

For simplicity, the shear modulus is obtained in the form:

$$
G=\frac{3(1-2 v)}{2(1+v)} K
$$

by assuming a constant value of the Poisson's ratio, $v=0.3$.

If the soil element is free to deform, no shear strains and consequently no shear stresses are developed. Under a 1-D constraint, when only vertical deformation is allowed, a shear strain must accompany the volumetric strain, so that the following lateral constraint is complied with:

$$
\dot{\varepsilon}_{h}=0
$$

By means of simple substitution, the lateral effective stress can be obtained from the previous equations in the form:

$$
\dot{\sigma}_{h}^{\prime}=\dot{s}-\frac{2}{3} G \dot{\varepsilon}_{v o l}=\dot{s}+\frac{2}{3} G \frac{\dot{e}_{w}}{1+e_{w}}
$$

It is worth noting that the change in total stress due to the water mass change during evaporation is disregarded in the formulation. The choice is justified by the fact that the latter term is two orders of magnitude smaller than the corresponding changes in suction. The total horizontal stress increment is given by:

$$
\dot{\sigma}_{h}=\dot{\sigma}_{h}^{\prime}-\dot{s}=\frac{2}{3} G \frac{\dot{e}_{w}}{1+e_{w}}
$$

During evaporation, the horizontal stress decreases as tensile stresses are developed. In the absence of weak spots, the idealised crack initiation condition can be defined as the moment when the total tensile stress attains the same value as the tensile strength:

$$
\left|\sigma_{h}\right|=\left|\sigma^{T}\right|
$$

The choice for an assessment in terms of total stresses is justified by the available experimental data on tensile strength, which is expressed in terms of the total stress needed to trigger tensile failure.

\subsection{Constitutive assumptions}

The response of the soil to a given change in water ratio in the simplified 1-D model depends on: (i) tensile strength; (ii) tensile stiffness; and (iii) soil water retention properties, which link the water ratio to the suction.

The response of the soil layer is analysed first at an elementary level (Model I), assuming constant values for the soil stiffness and strength. This approach has been used in previous models that have focussed exclusively on the crack onset $[3,6]$. In the second analysis (Model II), the stiffness and the strength are assumed to depend on the water ratio, which is in accordance with the evidence of current and previous experimental results [15]. Eventually, a dependence of the stiffness and water retention curve on the drying rate was considered, as well (Model III). It is worth reminding that no attempt was made to formalise the constitutive laws in a comprehensive thermo-mechanical framework. Instead, non-linear hypo-elastic laws were used, interpolating the experimental information.

The tensile strength was given a power law of the type:

$$
\sigma^{T}\left(e_{w}\right)=c e_{w}^{-d}
$$

The water retention curve was described by a standard van Genuchten's equation [16] with two independent parameters, assuming $m=1-1 / n$. The bulk modulus $\mathrm{K}\left(e_{w}\right)$ was calculated with Eq.(5) from the retention curve.

\section{Model predictions and discussion}

To investigate the consequences of the different assumptions on the clay layer response, a benchmark problem which replicates a typical clay board test in the laboratory was analysed.

\subsection{The benchmark problem}

A homogenous soil layer, $0.1 \mathrm{~m}$ thick, was assigned the initial state which characterised one of the laboratory tests. The water ratio profile was constant at the beginning of the tests, with a value of $e_{w 0}=2.55$. A final water content profile was assigned, based on laboratory measurements made after two weeks of drying. To simplify the analysis, a constant drying rate at each depth was assumed between the initial and final states. This allowed to define a set of water content values between the initial and final water content profiles, resulting in the water ratio evolution history in Fig. 2 . The water ratio rate varied from 0.03 to $0.01 d^{-1}$ with depth. Table 1 summarises the values of the parameters used in the simulations to describe the tensile strength (Eq.11). 
Table 1. Parameters used to describe tensile strength (Eq.11).

\begin{tabular}{|c|c|c|c|}
\hline Variable & $\begin{array}{c}\text { MODEL I } \\
\text { Constant }\end{array}$ & $\begin{array}{c}\text { MODEL II } \\
\text { Depending } \\
\text { on } e_{w}\end{array}$ & $\begin{array}{c}\text { MODEL III } \\
\text { Depending } \\
\text { on } e_{w}, \dot{e}_{w}\end{array}$ \\
\hline$\left|\sigma^{T}\right|(\mathrm{kPa})$ & $0.3 \mathrm{kPa}$ & $\begin{array}{c}\mathrm{c}=6.5 \mathrm{kPa} \\
\mathrm{d}=3.65\end{array}$ & $\begin{array}{c}\text { same as } \\
\text { model II }\end{array}$ \\
\hline
\end{tabular}

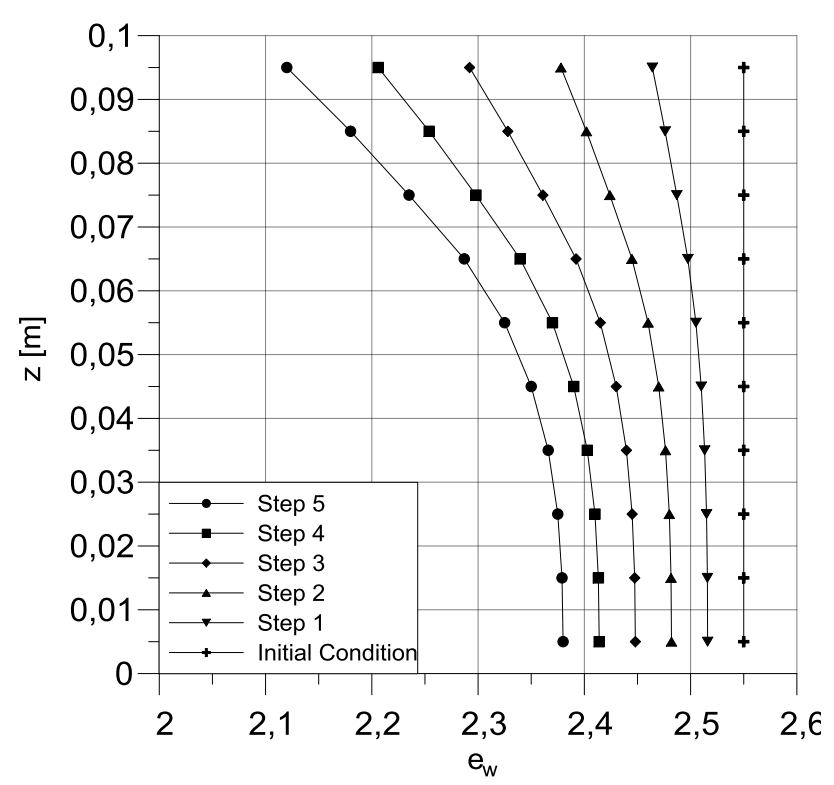

Figure 2. Water ratio profiles given as input to the 1-D model.

\subsection{Results}

In Fig. 3, the evolution of the suction profile is reported for Model II, showing an increase of the suction with time and attaining the maximum values at the top of the soil layer. As a consequence, the horizontal tensile stress also increases, reaching the highest value at the top of the layer (Fig. 4). Results for the simpler Model I, not shown, are qualitatively and quantitatively similar.

As depicted in Fig. 5, the tensile strength in Model II also increases during drying. In the figure, the constant average value assumed in Model I is represented by the dashed line. In spite of the tensile strength increase, the maximum tensile stress approaches the tensile strength and eventually reaches it (Fig. 6). For both Models I and II, the tensile strength is attained at the top of the layer before than at any other depth. This result is not due to the particular values assumed for the parameters, but it is a consequence of the shape of the curves, all having a similar dependence on the water ratio. If the tensile strength were to increase faster, no tensile crack would be predicted. On the contrary, if the condition for crack onset is met (Eq. 7), it would initially occur at the surface of the layer.

\subsection{Dependence on the rate of water ratio}

The results shown in Fig. 6 indicated that the dependence of the relevant quantities on the water ratio, as calibrated on the experimental results, was not enough to justify the occurrence of first cracks below the surface of the clay layer. Therefore, the crack initiation below the surface must be the result of some previously unaccounted factors.

The experimental data collected by Tollenaar [14] and previous research suggest that strength and stiffness might depend on the rate of change in water ratio, besides the water ratio. Nonetheless, while the rate of drying is relevant for the stiffness, the tensile strength is almost insensitive to it.

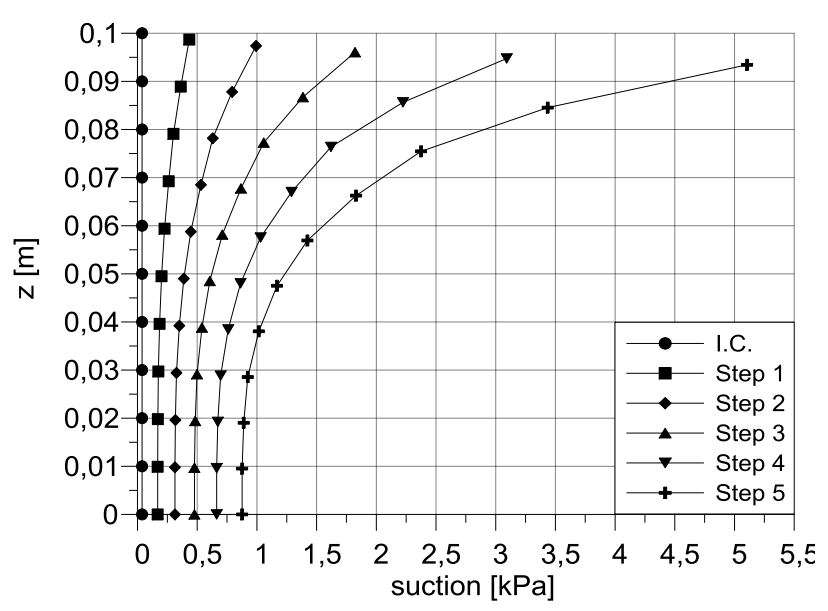

Figure 3. Suction profiles predicted by Model II.

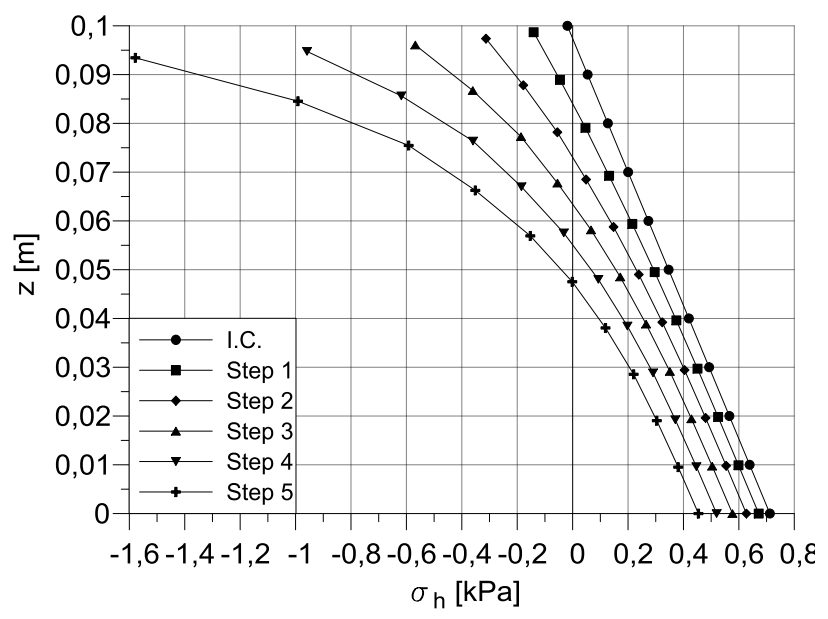

Figure 4. Horizontal total stress profile predicted by Model II.

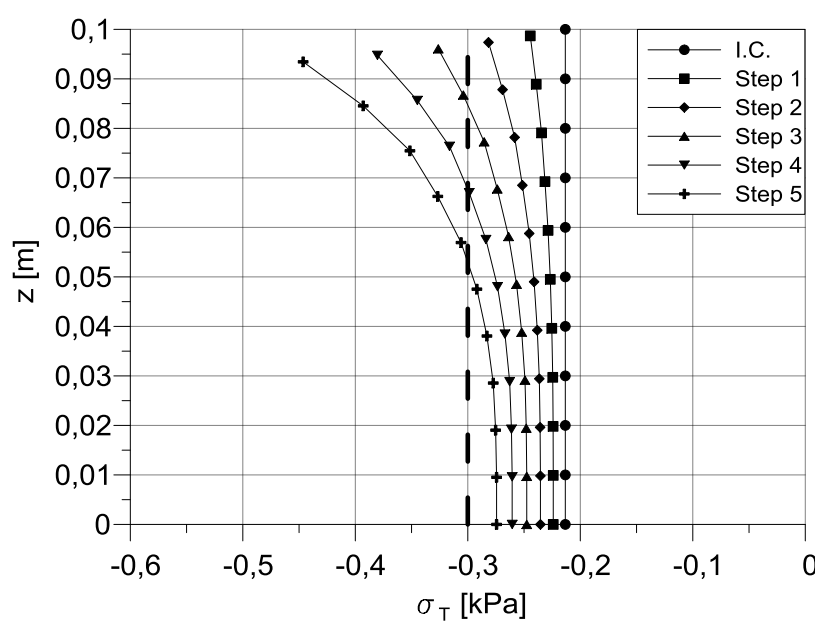

Figure 5. Tensile strength profile predicted by Model II. 


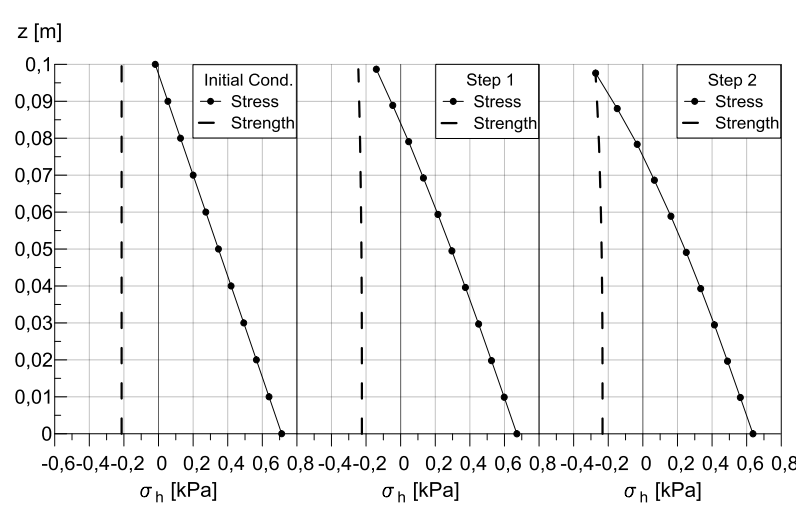

Figure 6. Comparison between the evolution of the horizontal stress and the tensile strength predicted by Model II.

Sensitivity analyses showed that the suction is the dominant factor in the shape of the horizontal stress with depth. To allow for the shape of the horizontal stress to change, the water retention curve has to be made dependent on the water ratio rate. Although this is not a common assumption, some previous work has already highlighted that this dependence may exist, either from a theoretical standpoint, or from experimental observations.

Experimental observations by Tollenaar [14] on the dependence of the tensile stiffness on the drying rate suggested that an intrinsic time scale characterises the response of the soil fabric to water content changes. A decrease in tensile stiffness at increasing drying rate can only be justified by the development of lower suction values. These lower suction values for a given water content suggest that fast drying tends to maintain an open fabric with larger pores. If drying is slow enough for the fabric to rearrange, a more massive fabric with smaller pores can develop as a consequence.

Based on this hypothesis, the water retention curve was made dependent on the rate of water ratio, by assuming a shape parameter $n$ of the form:

$$
n=\bar{n}+\bar{\eta} \dot{e}_{w}
$$

The correspondent curves are represented in Fig. 7, where the one independent from the drying rate (Model I and Model II) is sketched with a dashed line. The parameters used for the water retention curve are summarised in Table 2. In the range of drying rates analysed, $n$ is always higher than 1 . The corresponding tangent bulk moduli are shown in Fig. 8 .

Table 2. Parameters for the SWRC for Models I, II and III

\begin{tabular}{|c|c|c|c|}
\hline Parameter & $\begin{array}{c}\text { MODEL I } \\
\text { Constant }\end{array}$ & $\begin{array}{c}\text { MODEL II } \\
\text { Depending } \\
\text { on } e_{w}\end{array}$ & $\begin{array}{c}\text { MODEL III } \\
\text { Depending } \\
\text { on } e_{w}, \dot{e}_{w}\end{array}$ \\
\hline$\alpha\left(\mathrm{kPa}^{-1}\right)$ & 1.0 & 1.0 & 1.0 \\
\hline$n$ & 1.04 & 1.04 & $\begin{array}{c}\bar{n}=0.96 \\
\bar{\eta}=5.04(\mathrm{~d})\end{array}$ \\
\hline
\end{tabular}

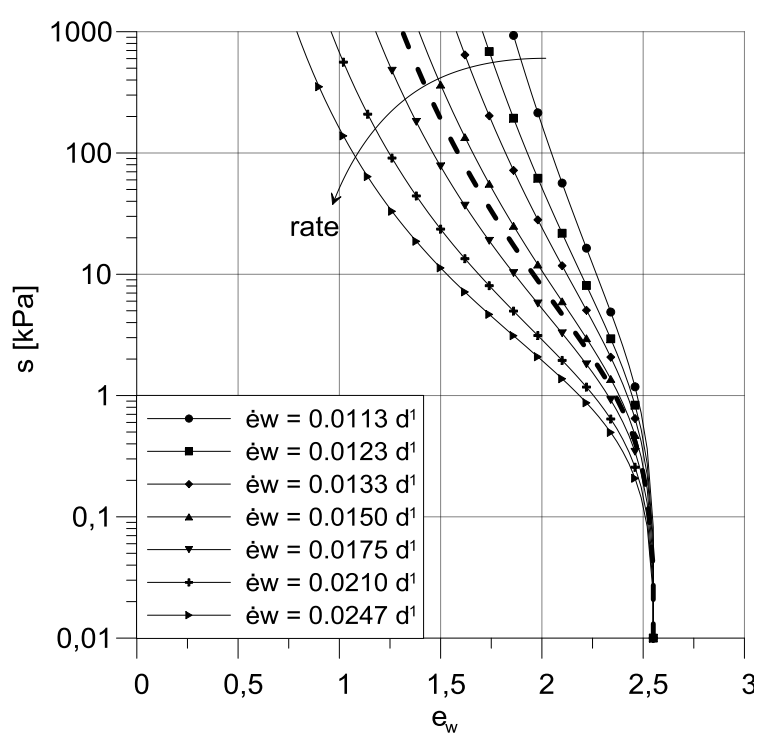

Figure 7. Suction vs water ratio and water ratio rate.

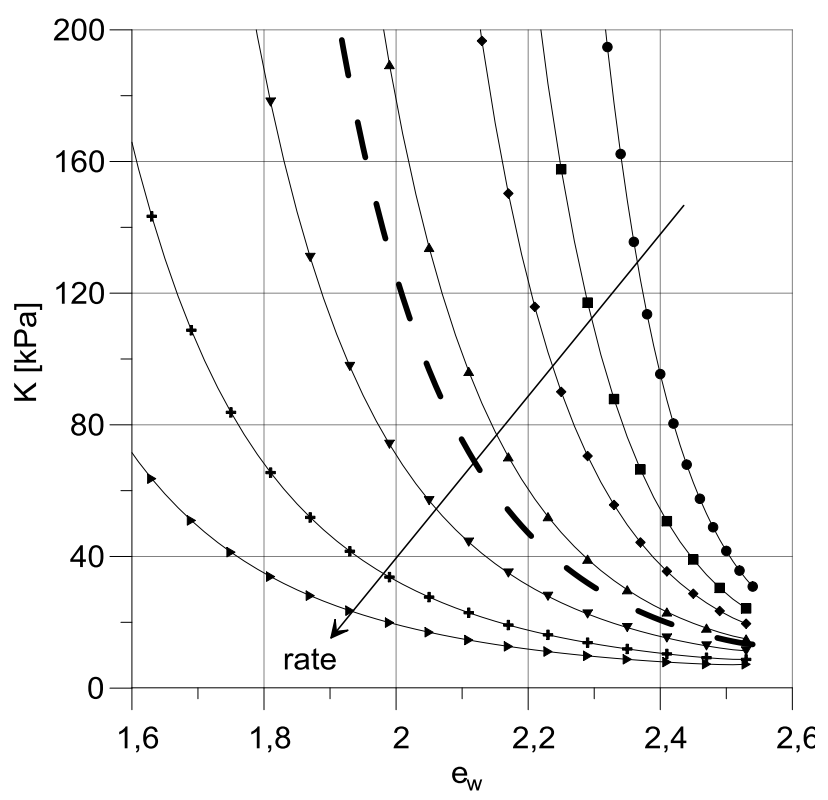

Figure 8. Bulk modulus vs water ratio and water ratio rate.

The drying rate dependence of the suction developed for a given water ratio yields a suction increase rate which is different for each depth. The implications are evident from the results of the simulation completed with Model III (Fig. 9).

The different rates at the different depths change the shape of the suction profile in time. As a result, the horizontal stress profile varies with depth, and the horizontal stresses at the top of the layer can increase less rapidly than at depth. As observed in Fig. 9, the onset of cracking is predicted for a water ratio profile occurring at the end of step 5. The critical depth is not on the surface anymore. On the contrary, the horizontal stress can reach the values of tensile strength at different depths below the surface, or even near to the bottom of the layer.

The results of this numerical simulation provide a demonstration of the possible theoretical development of cracks below the surface which were actually observed in the laboratory on the physical models. 


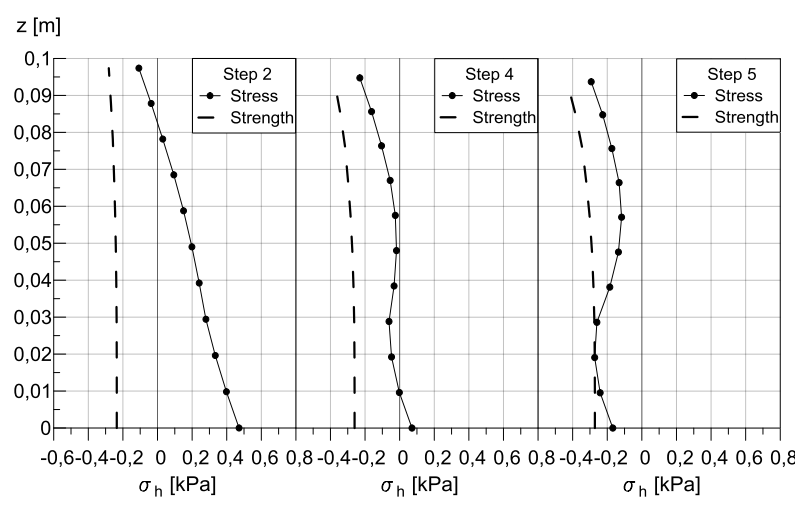

Figure 9. Comparison between the evolution of the horizontal stress and the tensile strength predicted by Model III.

\section{Conclusions}

The simple 1-D model for crack development was conceived to explore the consequences of various material behaviour assumptions on the response of a deformable clay layer to atmospheric desiccation. The work was motivated by the dominant role played by crack development in the mechanical and hydraulic behaviour of clay soils in engineering. Experimental observations in the laboratory have shown that cracks can begin and propagate below the surface of a desiccating soil layer. The observation was also made on clay boards specially designed to limit the influence of boundary constraints on the results of the test.

The simple models available in the literature were unable to explain the observation. This preliminary systematic study was aimed at investigating the necessary requirements for cracks to start at depth in shrinking clay, assuming the absence of weak spots and with a limited influence of external constraints.

Based on the model developed, a dependence of the water retention curve on the rate of drying seems to be the key feature necessary to reproduce the experimental evidence. This is a factor not considered in previous models for cracking. The results suggest that an intrinsic time scale for the mechanical response must be included with all the other factors being investigated. In a compressible soil, the dependence of the drying branch of the water retention curve on the rate of drying could be justified by a rate dependent fabric evolution.

\section{Acknowledgments}

The authors at the Delft University of Technology acknowledge the financial support of the Technologiestichting STW (NL) through the project Liftup-Lowlands (project 11344). N. Valimberti acknowledges the support of the Politecnico di Milano for his study exchange period at the Delft University of Technology. Dr. Caruso is acknowledged for his help in designing the clay board experiments at the Politecnico di Milano.

\section{References}

1. R. Weinberger, Geol. Soc. Am. Bull. 113(1), 20-31 (2001)

2. R. Weinberger, J. Struct. Geol. 21(4), 379-386 (1999)

3. P. H. Morris, J. Graham, D. J. Williams, Can. Geotech. J. 29(2), 263-277 (1992)

4. A. N. Abu-Hejleh, D. Znidarcic, J. Geotech. Eng. 121(6), 493-502 (1995)

5. J. M. Konrad, R. Ayad, Can. Geotech. J. 34(4), 477488 (1997)

6. H. Péron, L. B. Hu, L. Laloui, T. Hueckel, Third Asian Conference on Unsaturated Soils, 391-396 (2007)

7. A. Corte, A. Higashi, Technical report, U.S. Army snow and ice and permafrost research establishment (1960)

8. J. K. Kodikara, S. L. Barbour, D. G. Fredlund, Asian Conference on Unsaturated Soils, UNSAT ASIA 2000, 90, 693-698 (2000)

9. H. Nahalawi, J. K. Kodikara, Geol. Geotech. Eng. 24(6), 1641-1664 (2006)

10. C. S. Tang, B. Shi, C. Liu, L. Zhao, B. Wang, Eng. Geol. 101(3), 204-217 (2008)

11. C. S. Tang, Y. J. Cui, A. M. Tang, B. Shi, Eng. Geol. 114(3), 261-266 (2010)

12. S. Costa, J. Kodikara, B. Shannon, Geotechnique 63(1), 18-29 (2013)

13. S. B. Tamrakar, T. Mitachi, Y. Toyosawa, Experimental Unsaturated Soil Mechanics 207-218 (2007)

14. R. N. Tollenaar, PhD dissertation, in preparation (2016)

15. H. Trabelsi, M. Jamei, H. Zenzri, S. Olivella, Int. J. Numer. Anal. Meth. Geomech. 36(11), 1410-1433 (2012)

16. M. Van Genuchten, Soil Sci. Soc. Am. J. 44(5), 892898 (1980) 